\title{
A novel DNA methylation signature is associated with androgen receptor activity and patient prognosis in bone metastatic prostate cancer
}

\author{
Erik Bovinder Ylitalo ${ }^{1 \dagger}$, Elin Thysell ${ }^{1 \dagger}$, Mattias Landfors ${ }^{1 \dagger}$, Maria Brattsand ${ }^{1}$, Emma Jernberg ${ }^{1}$, Sead Crnalic ${ }^{2}$,
} Anders Widmark ${ }^{3}$, Magnus Hultdin ${ }^{1}$, Anders Bergh', Sofie Degerman ${ }^{1,4 \dagger}$ and Pernilla Wikström ${ }^{1 *+}$ (1)

\begin{abstract}
Background: Patients with metastatic prostate cancer (PC) are treated with androgen deprivation therapy (ADT) that initially reduces metastasis growth, but after some time lethal castration-resistant PC (CRPC) develops. A better understanding of the tumor biology in bone metastases is needed to guide further treatment developments. Subgroups of PC bone metastases based on transcriptome profiling have been previously identified by our research team, and specifically, heterogeneities related to androgen receptor (AR) activity have been described. Epigenetic alterations during PC progression remain elusive and this study aims to explore promoter gene methylation signatures in relation to gene expression and tumor AR activity.

Materials and methods: Genome-wide promoter-associated $\mathrm{CpG}$ methylation signatures of a total of 94 tumor samples, including paired non-malignant and malignant primary tumor areas originating from radical prostatectomy samples $(n=12)$, and bone metastasis samples of separate patients with hormone-naive $(n=14)$, short-term castrated $(n=4)$ or CRPC $(n=52)$ disease were analyzed using the Infinium Methylation EPIC arrays, along with gene expression analysis by Illumina Bead Chip arrays $(n=90)$. AR activity was defined from expression levels of genes associated with canonical AR activity.

Results: Integrated epigenome and transcriptome analysis identified pronounced hypermethylation in malignant compared to non-malignant areas of localized prostate tumors. Metastases showed an overall hypomethylation in relation to primary PC, including CpGs in the $A R$ promoter accompanied with induction of $A R$ mRNA levels. We identified a Methylation Classifier for Androgen receptor activity (MCA) signature, which separated metastases into two clusters (MCA positive/negative) related to tumor characteristics and patient prognosis. The MCA positive metastases showed low methylation levels of genes associated with canonical AR signaling and patients had a more favorable prognosis after ADT. In contrast, MCA negative patients had low AR activity associated with hypermethylation of ARassociated genes, and a worse prognosis after ADT.
\end{abstract}

\footnotetext{
*Correspondence: pernilla.wikstrom@umu.se

'Erik Bovinder Ylitalo, Elin Thysell and Mattias Landfors have contributed equally as first authors, and Sofie Degerman and Pernilla Wikström have contributed equally as last authors

1 Department of Medical Biosciences, Pathology, Umeå University, Umeå, Sweden

Full list of author information is available at the end of the article
}

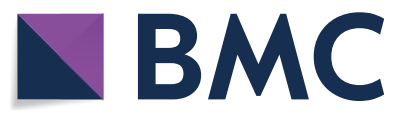

(c) The Author(s) 2021. Open Access This article is licensed under a Creative Commons Attribution 4.0 International License, which permits use, sharing, adaptation, distribution and reproduction in any medium or format, as long as you give appropriate credit to the original author(s) and the source, provide a link to the Creative Commons licence, and indicate if changes were made. The images or other third party material in this article are included in the article's Creative Commons licence, unless indicated otherwise in a credit line to the material. If material is not included in the article's Creative Commons licence and your intended use is not permitted by statutory regulation or exceeds the permitted use, you will need to obtain permission directly from the copyright holder. To view a copy of this licence, visit http://creativecommons.org/licenses/by/4.0/. The Creative Commons Public Domain Dedication waiver (http://creativeco mmons.org/publicdomain/zero/1.0/) applies to the data made available in this article, unless otherwise stated in a credit line to the data. 
Conclusions: A promoter methylation signature classifies PC bone metastases into two groups and predicts tumor AR activity and patient prognosis after ADT. The explanation for the methylation diversities observed during PC progression and their biological and clinical relevance need further exploration.

Keywords: Prostate cancer, Metastasis, Subtypes, MetA, MetB, MetC, DNA methylation, Gene expression, Androgen receptor, Prognosis

\section{Introduction}

Prostate cancer (PC) is a very common malignancy and a major cause of cancer mortality in men worldwide (https://gco.iarc.fr). Most patients with lethal PC develop bone metastases, which are treated with androgen deprivation therapy (ADT). Initially, ADT reduces metastasis growth, but eventually castration-resistant PC (CRPC) develops. Several treatment strategies for metastatic CRPC exist, with the majority aiming at inhibiting androgen receptor (AR) signaling [1]. Patients with CRPC show diverse responses to AR-inhibiting drugs, ranging from a strong response to complete resistance, underlining the need for complementary/alternative treatments. To guide future therapeutic developments, the tumor biology of metastatic PC must be understood in more detail.

The genetics behind CRPC have been comprehensively explored [2-5], indicating $A R$ amplification as the most common event (seen in about $50 \%$ of the cases). Also, structural $A R$ rearrangements and $A R$ mutations are frequently observed together with mutations in cofactors regulating the transcriptional activity of the AR. The prognostic and therapy-predicting value of those genetic defects in relation to AR targeting therapies is still unclear $[5,6]$.

By exploring the gene expression in clinical samples of PC bone metastases, we have identified transcriptomic as well as proteomic profiles of suggested clinical relevance [7-12]. Specifically, we have identified three molecular subtypes of bone metastases, termed MetA-C, based on the diverged expression of genes related to AR activity, cell cycle activity, and stroma response [12]. The MetA subtype shows high AR activity in comparison to MetB and $C$, while MetB shows higher cell cycle activity than the other subtypes. Accordingly, MetA patients have the best prognosis after ADT, while MetB patients have by far the worst prognosis. Interestingly, the MetA-C subtypes could be intrinsic, as selected features of MetB (low AR activity and high proliferation) were traceable back to the corresponding primary tumors [12]. In line with this, PC subtypes with characteristics similar to the MetA-C subtypes have been identified by transcriptomic analysis of primary prostate tumors [13, 14].

The mechanistic explanation for the development of different metastasis subtypes is unknown. The subtypes could relate to diverse basal/luminal cellular origin, genetic defects as described above, but also to epigenetic defects affecting differentiation and clonal expansion, possibly influenced by the metastatic microenvironment and/or by therapy. Alterations in DNA methylation have been described to contribute to PC development and progression into metastatic disease [15-17]. The aim of the current study was to explore the promoter DNA methylation pattern in relation to gene expression during PC disease progression. Specific interest was put into analyzing promoter methylation levels in relation to canonical AR activity in metastasis samples and to patient prognosis.

\section{Material and methods \\ Patients}

Samples of bone metastases were obtained from a series of fresh-frozen biopsies collected from 70 patients with PC operated for metastatic spinal cord compression at Umeå University Hospital (2003-2013). The patient series have been previously described $[7,9,12]$ and clinical characteristics of patients included in the current study are summarized in Table 1 . The study also included 12 separate patients with localized PC who were treated with radical prostatectomy at Umeå University Hospital, between 2008 and 2009; mean age for these men was 60 years (range 48-68 years) and mean serum level of prostate specific antigen (PSA) was $11 \mathrm{ng} / \mathrm{ml}$ (range $3.5-26 \mathrm{ng} / \mathrm{ml})$. Clinical local stage was T2 $(n=4)$ or T3 $(n=8)$ and Gleason score (GS) was $7(n=10)$ or $8(n=2)$.

\section{Tissue sample preparation}

Bone metastasis samples were instantly fresh-frozen in liquid nitrogen. Fresh radical prostatectomy specimens were received at the pathology department immediately after surgery and cut into $0.5 \mathrm{~cm}$ thick slices. From these slices, 20 samples were taken using a $0.5 \mathrm{~cm}$ skin punch and frozen in liquid nitrogen within $30 \mathrm{~min}$ after surgery. The prostate slices were formalin-fixed, embedded in paraffin, cut in $5 \mu \mathrm{m}$ sections, whole-mounted, and stained with hematoxylin-eosin. Tissue sample composition (non-malignant or malignant) of the frozen pieces was determined from their location in the whole-mount sections.

Representative areas of fresh-frozen bone metastasis (M) samples, non-malignant $(\mathrm{N})$ and malignant tumor 
Table 1 Clinical characteristics of patients with prostate cancer from whom samples of bone metastases were analyzed by Infinium Methylation EPIC arrays

\begin{tabular}{|c|c|c|c|}
\hline Clinical characteristics & $\begin{array}{l}\text { Hormone-naíve } \\
n=14\end{array}$ & $\begin{array}{l}\text { Short-term } \text { castrated }^{a} \\
n=4\end{array}$ & $\begin{array}{l}\text { Castration-resistant }{ }^{b} \\
n=52\end{array}$ \\
\hline Age at diagnosis (years) & $78(74-80)$ & $75(72-76)$ & $71(64-75)$ \\
\hline Age at metastasis surgery (years) & - & - & $73(68-80)$ \\
\hline Serum PSA at diagnosis (ng/ml) & $320(82-980)$ & $2300(560-4300)$ & $82(40-510)$ \\
\hline Serum PSA at metastasis surgery $(\mathrm{ng} / \mathrm{ml})$ & - & - & $250(82-630)$ \\
\hline \multicolumn{4}{|l|}{ Bicalutamide prior to metastasis surgery ${ }^{c}$} \\
\hline Yes & 0 & 0 & 28 \\
\hline No & 14 & 4 & 24 \\
\hline \multicolumn{4}{|l|}{ Radiation prior to metastasis surgery ${ }^{d}$} \\
\hline Yes & 0 & 0 & 7 \\
\hline No & 14 & 4 & 45 \\
\hline \multicolumn{4}{|l|}{ Chemotherapy prior to metastasis surgery } \\
\hline Yes & 0 & 0 & 9 \\
\hline No & 14 & 4 & 43 \\
\hline \multicolumn{4}{|l|}{ Ra-223 prior to metastasis surgery } \\
\hline Yes & 0 & 0 & 6 \\
\hline No & 14 & 4 & 46 \\
\hline
\end{tabular}

Continuous values are given as median (25th; 75th percentiles)

${ }^{\text {a }}$ Short-term treated patients had received androgen ablation therapy for 1-3 days before metastasis surgery

${ }^{b}$ Castration-resistant patients had disease progression after long-term androgen deprivation therapy. First line androgen-deprivation therapy (ADT) includes surgical ablation, LHRH/GNRH agonist therapy, and therapy with anti-androgens

${ }^{c}$ Castration therapy as stated above and bicalutamide for treatment of CRPC

${ }^{\mathrm{d}}$ Radiation towards operation site

(T) areas, and prostatectomy tissue samples were cryosectioned into extraction tubes. The fraction of epithelial cells in the samples was determined by examination of parallel hematoxylin-eosin-stained sections.

\section{DNA methylation profiling}

Genomic DNA was isolated by the AllPrep DNA/RNA/ Protein method (Qiagen, Hilden, Germany) and DNA quality and quantity were determined by spectrophotometry (NanoDrop, Thermo Scientific, Wilmington, DE, USA) and the Qubit dsDNA BR assay kit on a Qubit 3.0 Fluorometer (Invitrogen, Carlsbad, CA). DNA (300 ng) was bisulfite converted using the EZ DNA Methylation Kit (Zymo Research, Irvine, USA) and thereafter applied to the Infinium Methylation EPIC arrays (lllumina, San Diego, CA), and operated according to the manufacturer's instructions. Array analysis including pre-processing and normalization was performed as previously described [18], with some modifications. The quality of each array was evaluated with built-in controls and the matching identities of the $\mathrm{N}$ and $\mathrm{T}$ paired samples were confirmed by using the 59 built-in single nucleotide polymorphisms (SNPs) (Additional file 1: Fig. S1).

The fluorescence intensities were extracted using the Methylation Module (1.9.0) in the Genome Studio software (V2011.1), whereas pre-processing and downstream analysis was done using $\mathrm{R}$ (v3.4.1). An overview of the pre-processing steps is shown in Additional file 2: Fig. S2. Data was normalized using the BIMQ method to compensate for the two different bead types used in the array [19]. Cross-reactive CpG probes that aligned to multiple loci in the genome or were located in methylation quantitative trait loci (meQTLs) $[20,21]$ or located less than 5 bp from a known single nucleotide polymorphism in the European population [22] were excluded. CpG probes with detection $P$ value $>0.05$ in any sample were also excluded. The methylation level ( $\beta$-value) of each CpG site ranging from 0 (no methylation) to 1 (complete methylation) was used as the measure for methylation level in down-stream analyses.

Methylation levels ( $\beta$-values) were extracted for promoter associated CpGs located in the TSS1500 (the region that covers -200 to -1500 nucleotides upstream of Transcription Start Site (TSS)), TSS200 (from TSS to -200 nucleotides upstream of TSS), and $5^{\prime}$ UTR regions, which showed an overall $\mathrm{SD}>0.05$. Differentially methylated $\mathrm{CpG}$ sites (DM-CpGs) were defined as a mean delta- $\beta$-value $>0.3$ or $<-0.3$ between compared groups. Heatmaps were produced in R (v3.4.1) without scaling and using default settings for clustering. 
Principal component analysis (PCA), based on centralized $\beta$-values were used for unsupervised multivariate projection.

\section{Copy number variation analysis}

The raw signal intensity data from the HumanMethylationEPIC arrays was imported to $\mathrm{R}$ by the minfi package [23] and CNV analysis was performed using the conumee package [24]. The 12 non-malignant samples were used as reference samples and the analysis included the $A R$ gene as a detailed region. The CNV status of AR was determined through manual inspection. Human genome GRCh37 (NCBI)/hg19 was used for assigning all chromosome positions.

\section{Whole genome expression analysis}

The majority (96\%) of the samples applied to DNA methylation analysis had been previously applied to whole genome expression array analysis using the human HT12 Illumina Beadchip technique (Illumina, San Diego, CA) $[7,9]$. In 48 cases, total RNA was extracted in parallel with the DNA extractions using the AllPrep DNA/ RNA/Protein method. In the other cases, RNA had been extracted from parallel tissue sections using the AllPrep or the Trizol method (Invitrogen, Carlsbad, CA). Bead chip data from two separate gene expression studies (GEO Datasets GSE29650 and GSE101607) were combined for all probes with average signals above two-times the mean background level in at least one sample per study array, leaving 15,232 gene transcripts for subsequent analysis. The gene expression arrays were individually normalized using the quantile method and data was centered by the mean for each probe. Gene transcripts were matched to corresponding promoter CpG sites by Refseq accession and Entrez gene identification numbers.

\section{Assessment of AR activity and proliferation in metastasis samples}

The AR activity of a metastasis sample was defined by its relative expression levels of genes predefined to be associated with canonical AR activity; AR, FOXA1, HOXB13, KLK2, KLK3, NKX3-1, STEAP2, and TMPRSS2, as previously described [9]. The AR activity of each metastasis sample was represented by its score on the first PCA score vector $(t[1])$, capturing the largest variation in the data as a linear combination of the selected transcript levels. Similarly, tumor cell proliferation in metastasis samples was represented by the $t[1]$ scores obtained from PCA of genes predefined to be associated with cell cycle proliferation [25]; RAD54L, CDC20, CENPF, CDKN3, PBK, TOP2A, LCMT2, ASF1B, KIF2OA, CDCA8, NUSAP1, PRC1, PLK1, CDCA3, CEP55, CDC2, KIF11,
BUB1B, TK1, ASPM, PTTG1, ORC6L, FOXM1, RAD51, CENPM, CDAN1, KIAA0101, MCM10.

\section{Classification of metastasis samples into metastasis subtypes}

The metastasis samples were classified into molecular metastasis subtypes MetA $(n=46), \operatorname{MetB}(n=12)$, and MetC $(n=8)$ by PCA and unsupervised clustering based on their transcription profiles, as previously described [12]. Four samples were not possibly to classify in relation to MetA-C, due to lack of transcriptomic data.

\section{Functional enrichment analysis}

Functional enrichment analysis was performed by the MetaCore software (GeneGo, Thomson Reuters, New York, NY). In comparisons between different groups ( $\mathrm{T}$ and $\mathrm{N}$ or $\mathrm{M}$ and $\mathrm{T}$ ), analysis was based on genes showing (i) DM-CpGs (mean delta- $\beta$-value $>0.3$ or $<-0.3$ in promoter regions), (ii) inverse correlation between the methylation levels ( $\beta$ values) of CpG sites (mCpGs) and the corresponding transcript levels, and iii) significantly different transcript levels $(P<0.05)$. In the analysis of promoter methylation levels in relation to AR activity, analysis was based on genes showing (i) positive or negative correlations between $\mathrm{mCpGs}(\beta$ values) and AR activity score (corr $>0.4 ;-<0.4$ ), (ii) a considerable difference between samples (stdev $>0.15$ ), and (iii) inverse correlation ( $>0.4)$ between $\mathrm{mCpGs}(\beta$ values) and the corresponding transcript levels. Sets of genes associated with a functional pathway were determined as significantly enriched based on $P$ values representing the probability for a process to arise by chance, considering the numbers of enriched gene products in the data versus the number of genes in the process. $P$ values were adjusted by considering the rank of the process, given the total number of processes in the MetaCore ontology. Enriched pathways were expressed as pathway maps or as process networks, which were created by MetaCore on the basis of both pathway maps and gene ontology processes.

\section{Statistics}

The Wilcoxon rank sum test was used to compare independent groups and the Wilcoxon signed rank sum test for paired data. Bivariate correlations were analyzed according to Pearson. Survival analysis was performed by Kaplan-Meier analysis and by multivariate Cox regression analysis, with death of $\mathrm{PC}$ as event and death by other causes as censored events. The false discovery rate (FDR) were controlled according to the BenjaminiHochberg procedure. To evaluate the consistency of the clusters a silhouette analysis was performed using the cluster package [26] in R. 


\section{Results}

DNA promoter methylation associated with prostate cancer development and progression

The methylation levels ( $\beta$-values) of CpG sites (mCpGs) were analyzed in pairs of non-malignant $(\mathrm{N})$ and malignant primary tumor $(\mathrm{T})$ areas originating from radical prostatectomy samples of $12 \mathrm{PC}$ patients. In parallel, the $\mathrm{mCpG}$ profiles were analyzed in 70 bone metastasis (M) samples of separate patients with hormonenaive (HN, $n=14)$, short-term castrated (ST, $n=4)$, or castrate-resistant disease (CRPC, $n=52$ ) (Table 1 ). To facilitate correlation studies between methylation levels of gene promoter regions and corresponding transcript levels, the analysis was focused on CpG sites in promoter regions (defined as CpGs located within the TSS1500, TSS200, 5'UTR regions), leaving 121,944 sites for down-stream analysis after filtration (Additional file 2: Fig. S2).

Principal component analysis based on the 121,944 promoter-associated $\mathrm{mCpGs}$ showed a clear separation between the $\mathrm{N}, \mathrm{T}$, and $\mathrm{M}$ samples, except for two T and two $\mathrm{M}$ samples (Fig. 1A). By histological re-examination of tissue sections, the miss-classified samples could not be explained by any obvious morphological characteristics.

The epithelial cell fraction was estimated by microscopy examination of tissue sections. The $\mathrm{N}$ samples showed a significantly lower epithelial cell content compared to the $\mathrm{T}$ and $\mathrm{M}$ samples; median (25th; 75th percentiles) epithelial fraction in $\mathrm{N}, \mathrm{T}$, and $\mathrm{M}$ samples were 50 (30-50), 80 (70-80), and $70(50-80) \%$, respectively $(P<0.001$, Fig. 1B). The $\mathrm{M}$ samples did not cluster related to epithelial cell fraction (Fig. 1B) or previous treatment (Fig. 1A). Based on a difference in $\beta$-value of at least \pm 0.3 , a total of 15,358 DM-CpGs were found for T, N, and/or $\mathrm{M}$ samples (Fig. 1C).

Differential methylation analysis between $\mathrm{T}$ and $\mathrm{N}$ samples resulted in 4360 DM-CpGs, most of which (86\%) corresponding to CpG hypermethylation of the $\mathrm{T}$ samples (Fig. 1C). Those DM-CpGs (representing 1759 unique genes) clearly separated $\mathrm{T}$ and $\mathrm{N}$ samples in a hierarchical cluster analysis (Fig. 1D). The pronounced hypermethylation seen in the tumor samples was associated with significantly reduced gene expression levels in $25 \%(n=442)$ of the DM genes (Additional file 4: Table S1). Functional enrichment analysis indicated altered processes of potential importance for cellular transformation and cancer development, including e.g. regulation of proliferation, angiogenesis and cell adhesion (Additional file 5: Table S2). In contrast, genes showing hypomethylation in combination with significantly induced expression in primary PC $(n=67,4 \%$ of the DM genes) (Additional file 4: Table S1) showed no significant functional enrichment, according to the MetaCore software (Additional file 5: Table S2).

To further focus on DNA methylation alterations associated with progression from a primary tumor in the prostate to metastasis in the bone, differently methylated genes between these stages were identified. Among the 1183 DM-CpGs between $\mathrm{T}$ and $\mathrm{M}$, the majority (88\%) corresponded to hypomethylation of the M samples (Fig. 1C, E). Notably, only 70 of the hypomethylated genes also showed significantly increased transcript levels and for these no consensus regarding enriched ontologies could be found, while functional enrichment analysis of the few hypermethylated genes with parallel reduction in gene expression $(n=23)$ suggested altered apoptosis response in metastases compared to primary tumors (Additional file 5: Table S2 and Additional file 6: Table S3).

The fact that most of the DM-CpGs seen between M and $\mathrm{T}$ were also DM between $\mathrm{M}$ and $\mathrm{N}$, suggests that the overlapping $\mathrm{CpGs}$ were likely organ-related rather than associated with bone metastasis (Fig. 1C). Further analysis was therefore focused on the 85 DM-CpGs (80 unique genes) that uniquely diverged between $M$ and $T$ and possibly related specifically to disease progression (Figs. 1C, 2A). Of the 85 DM-CpGs between $\mathrm{M}$ and $\mathrm{T}$ samples, 94\% showed a general hypomethylation in the metastases (Figs. 1C, 2A). Hierarchical cluster analysis identified three main clusters with diversity in the degree of hypermethylation among the $M$ samples (Fig. 2A). These clusters did not correlate to treatment status, nor to epithelial cell fraction (data not shown). Next, an integrated transcriptome analysis that aimed to identifying hyper- or hypomethylated genes with differential gene expression of potential relevance for bone metastasis was performed. Corresponding transcript levels were available for 55 of the 80 unique differently methylated genes. A set of genes showing either hypermethylation in combination with significant gene expression downregulation $(S L C 8 B 1)$ or hypomethylation in combination with gene overexpression (HECW2, AR, GMNN, TSPAN18, and $M E F V$ ) in metastases (Fig. 2B) were identified. The hypomethylated ubiquitin ligase $H E C W 2$, the DNA replication inhibitor GMNN (geminin), and the tetraspanin TSPAN18 showed clear overexpression in metastases, and may deserve further studies, while fold changes for the innate immunity regulator $M E F V$ and the sodium/ calcium exchanger $S L C 8 B 1$ were less convincing. Of special notice was the hypomethylation of the $A R$ gene in the $\mathrm{M}$ samples. Although gene amplification and overexpression of the $A R$ in CRPC have been thoroughly described in the literature, less is known about its epigenetic regulation, and the $A R$ was therefore chosen for further exploration. 
A

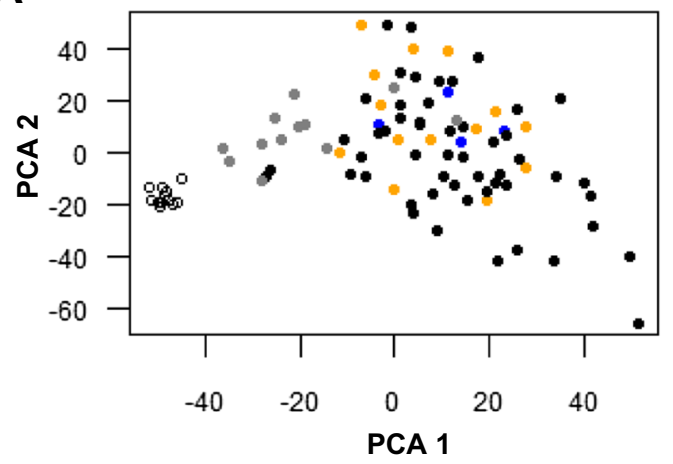

Non-malignant prostate tissue

Primary tumor

Hormone Naïve Metastasis

Short term treated Metastasis

CRPC Metastasis
B
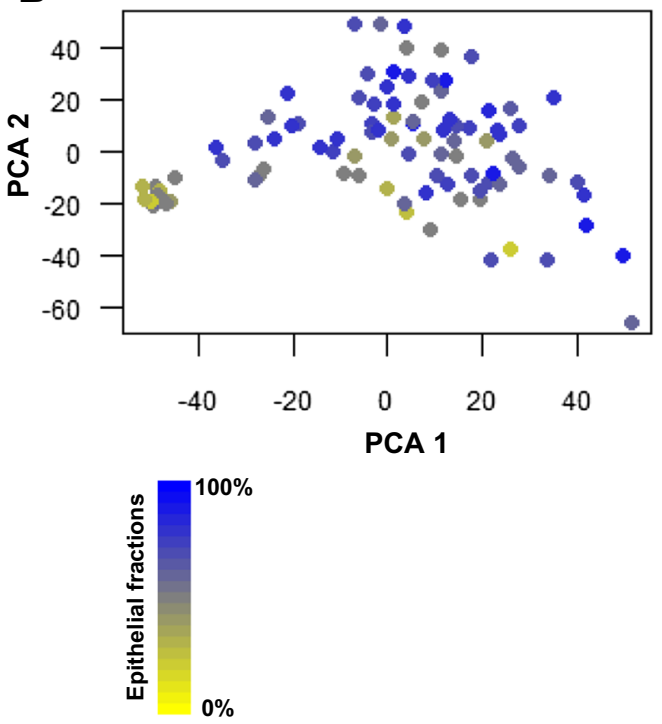

C

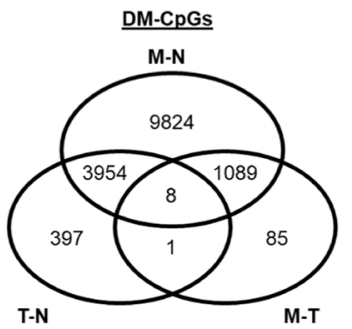

Hypermethylated DM-CpGs

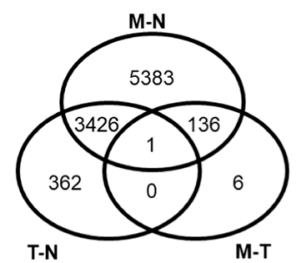

Hypomethylated DM-CpGs

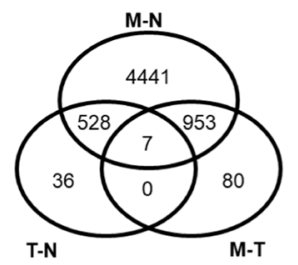

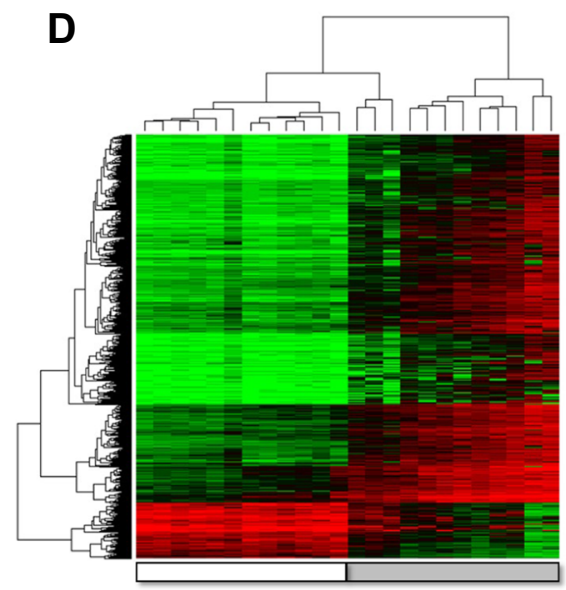
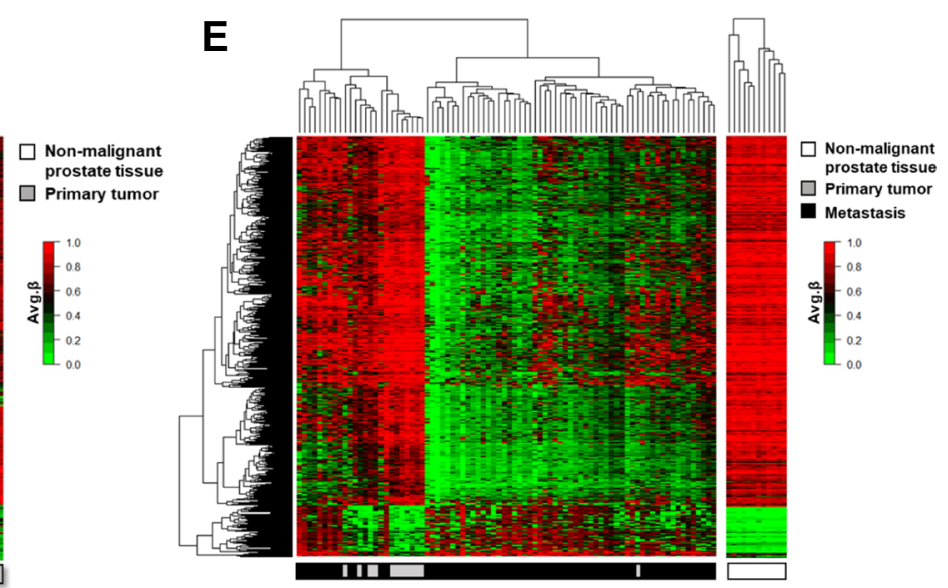

Fig. 1 Identification of differently methylated CpGs (DM-CpGs) in non-malignant prostate (N), primary prostate tumor ( $\mathrm{T}$ ), and bone metastasis (M) tissue samples. A, B PCA plots based on 121,944 promoter associated CpGs in the HumanMethylation EPIC arrays. A The colors represent patient treatment; no treatment/hormone naïve (orange), castrate-resistant prostate cancer (CRPC) (black), and short term androgen deprivation therapy (blue). Non-malignant (white) and primary tumor (grey) tissues are shown. B The same loading plot as in Fig. A with epithelial fractions shown. C DM-CpG sites between the primary tumor $(T)$ and non-malignant $(N)$ prostate tissue $(n=4360)$, between the $N$ and metastasis (M) tissue $(n=14,875)$ and between the M and T tissue $(n=1183)$. The overlaps include DM-CpGs both in the same direction between comparisons (e.g. hyper-hyper) as well as DM-CpGs in different directions (e.g. hypo-hyper). The hyper- and hypomethylated CpGs are also separately shown. D Heatmap showing the 4360 DM-CpGs (identified in Fig. C) between T $(n=12)$ and N $(n=12)$. E Heatmap showing the 1183 DM-CpGs (identified in Fig. C) between $\mathrm{M}(n=70)$ and $\mathrm{T}(n=12)$ 
A

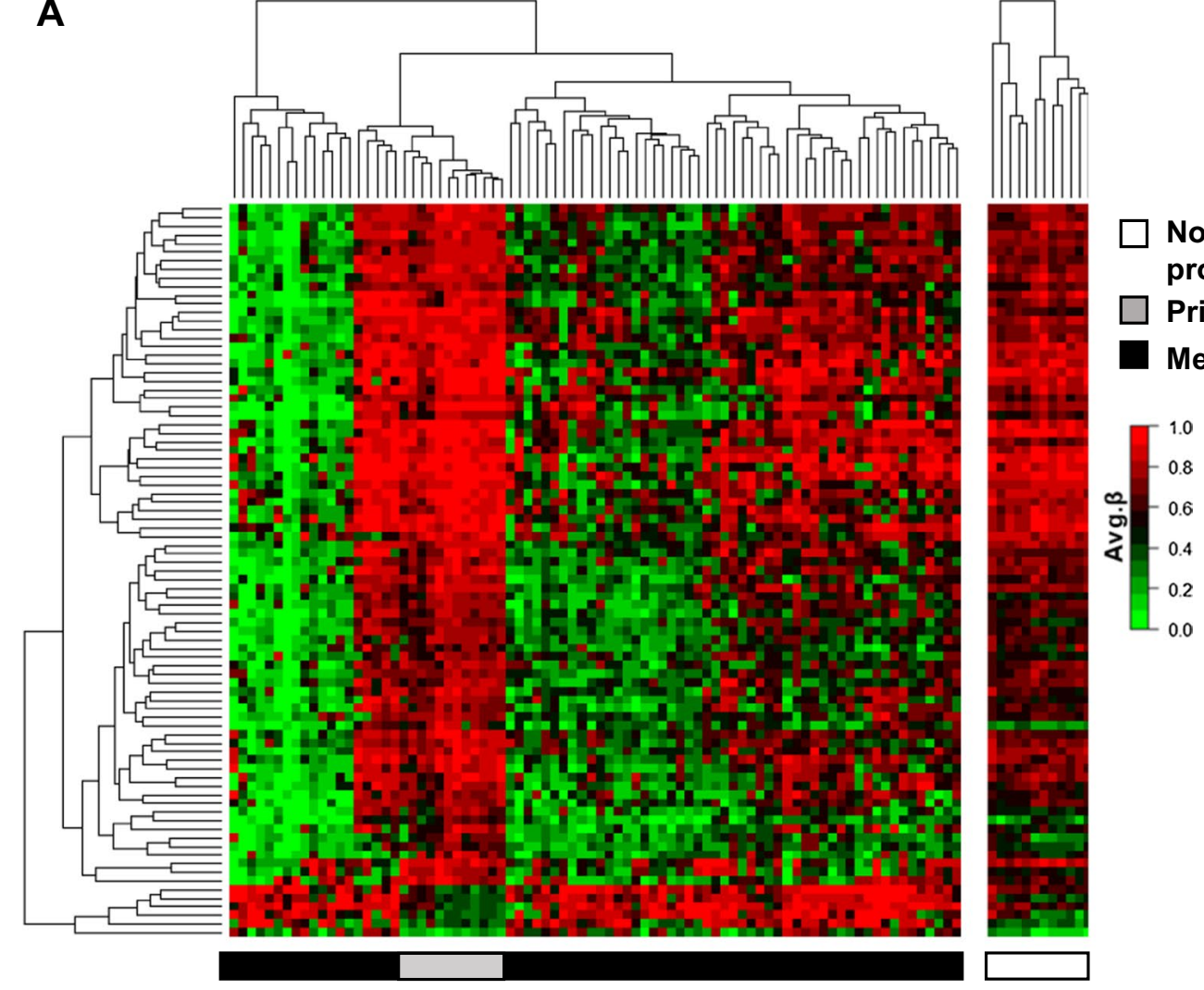

B

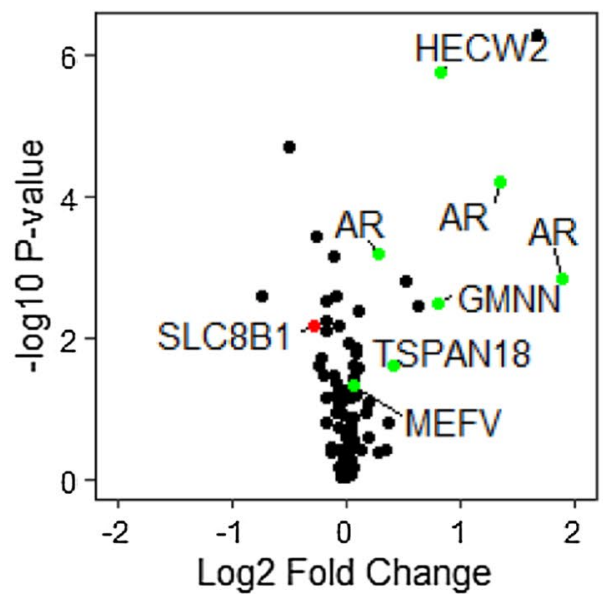

Fig. 2 Integrated analysis of promoter DNA methylation and gene expression in metastases and primary tumors. A Heatmap showing the 85 unique differently methylated CpGs (identified in Fig. 1C) between the primary tumors (T) $(n=12)$ and the bone metastases (M) $(n=70)$. B Vulcano plot of the corresponding gene expression. 6 unique genes that were either hypermethylated with decreased gene expression (red) or hypomethylated with increased gene expression (green) in metastasis compared to primary tumors are indicated. The AR is represented by 3 different gene probes in the Illumina BeadChip array data, corresponding to two AR transcripts (coding for the full-length AR and the AR45 splice variant)

Androgen receptor gene amplification, promoter DNA hypomethylation, and mRNA overexpression in metastases The $A R$ mRNA overexpression observed in the $\mathrm{M}$ samples (Fig. 2B) corresponded to two transcript variants (coding for the full-length AR and its shorter AR45 variant, lacking the $\mathrm{N}$-terminal domain). Accordingly, 
hypomethylation was observed at several $A R$ promoterassociated CpGs in $\mathrm{M}$ compared to $\mathrm{N}$ and $\mathrm{T}$ samples, with the most pronounced CpGs being located close to the TSS200 and 5'-UTR regions (Fig. 3A). In order to examine if $A R$ amplification, in addition to $A R$ promoter hypomethylation, could be associated with the high $A R$ mRNA levels observed, the $A R$ copy numbers of the M samples were assessed and found to be amplified in $64 \%$ of the cases. Metastases with $A R$ amplification showed the highest $A R$ mRNA levels, but also M samples with a normal $A R$ copy number had significantly higher expression levels than $\mathrm{N}$ and $\mathrm{T}$ samples (Fig. 3B). As seen in Fig. 3A, all but one of the untreated $M$ samples had a normal $A R$ copy number, while $A R$ promoter hypomethylation could be observed already at that stage. Interestingly, there was no difference in AR activity in-between M samples with and without $A R$ amplification, as determined by their score on a vector constructed from mRNA levels of genes involved in or regulated through canonical AR signaling (Fig. 3B). This indicates that AR activity within $M$ samples is regulated by other means than by AR copy number, AR promoter methylation, and AR expression levels only.

\section{DNA promoter methylation associated with tumor androgen receptor activity and patient prognosis}

To identify mCpGs related to the AR activity within PC metastases, and thus their potential AR dependence, we extracted data for all promoter CpG sites showing positive or negative correlation (correlation $\geq 0.4$, StDev $>0.15$ ) to the sample AR activity score. This Methylation Classifier for Androgen receptor activity (MCA) signature consisted of 2970 unique CpGs and separated the $\mathrm{M}$ samples into two main clusters named MCA negative and MCA positive (Fig. 4A). The frequency of $\mathrm{HN}$, ST or CRPC samples did not significantly differ between MCA negative and positive metastasis, and neither did the frequency of $A R$ amplified samples or the median epithelial cell fraction (Fig. 4A). Interestingly, the samples of the molecular metastasis subtype MetA were enriched in MCA positive metastasis, while MetB and MetC samples were enriched in MCA negative metastasis $(P<0.001$, Chi-square test).

MCA positive metastasis showed hypomethylation and overexpression of genes associated with recurrent gene fusions in $\mathrm{PC}$ and $\mathrm{AR}$ activation and downstream signaling in PC (Fig. 4A, Table 2), indicating high AR dependence in those cases. In contrast, MCA negative showed hypermethylation and low expression of many genes related to AR activity and instead hypomethylation and high expression of genes associated with epithelial to mesenchymal transition (EMT), cytoskeletal remodeling, and immune response (Fig. 4A, Table 2). Accordingly, MCA negative metastases had significantly lower AR activity scores and a tendency of higher proliferation scores than MCA positive metastases (Fig. 4B, C). In addition, MCA negative patients showed significantly lower serum PSA levels and a worse prognosis after ADT than MCA positive patients (Fig. 4D, E). The median cancer-specific survival after ADT was 30 months for MCA negative patients compared to 60 months for MCA positive patients $(P=0.001$, Fig. $4 \mathrm{E})$. Analysis of cluster consistency showed an average silhouette width of 0.23 in the MCA positive and negative clusters (Additional file 3: Fig. S3).

In Cox regression analysis, the MCA negative signature, proliferation score, and the MetB subtype were significantly associated with poor survival after ADT (Table 3). Multivariate Cox analysis indicated that the MCA signature provided prognostic information that was independent from the proliferation and subtype status, with an increased risk of 2.7 for patients being MCA negative $(P=0.0017)$ (Table 3$)$. No significant associations were observed between patient prognosis and AR activity score, $A R$ amplification status, serum PSA, or age (Table 3).

\section{Discussion}

This study integrates genome-wide promoter methylation data with gene expression data and describes consistent changes occurring during $\mathrm{PC}$ disease progression from non-malignant prostate epithelium to primary PC and further to bone metastatic disease. In metastatic samples, the study also specifically explores if promoter

\footnotetext{
(See figure on next page.)

Fig. 3 Promoter DNA methylation and gene expression of the androgen receptor (AR) during prostate cancer disease progression. A Methylation beta levels at individual CpGs in the AR promoter region for non-malignant $(N, n=12)$, primary tumor $(T, n=12)$ and metastasis samples with (M, $n=42$ ) and without ( $n=24)$ AR amplification, as well as mean N/T/M methylation levels. AR amplification status was defined according to copy number analysis performed based on signal intensity data from the HumanMethylationEPIC arrays, as described in the method section. Each CpG is shown as a dot, and specified in Additional file 7: Table S4. Red dots represent CpGs within the TSS200 region close to the two alternative transcription start sites, black dots represent CpGs within the TSS1500 promoter region and the green dots CpGs within the 5'UTR region. B Box plots of mean methylation beta values in the N/T/M group of the two differently methylated CpG (DM-CpG) sites (IIlumina ID cg01218690 and cg13873881) within the AR. C Relative AR mRNA levels (transcript variant corresponding to full length AR) and AR activity scores in the corresponding tissue samples, calculated based on Illumina Bead Chip arrays data as described in the methods section and analyzed in relation to the $A R$ amplification status given in $3 \mathrm{~A} .{ }^{* * *} P<0.001, \#=\mathrm{DM}-\mathrm{CpG}$
} 

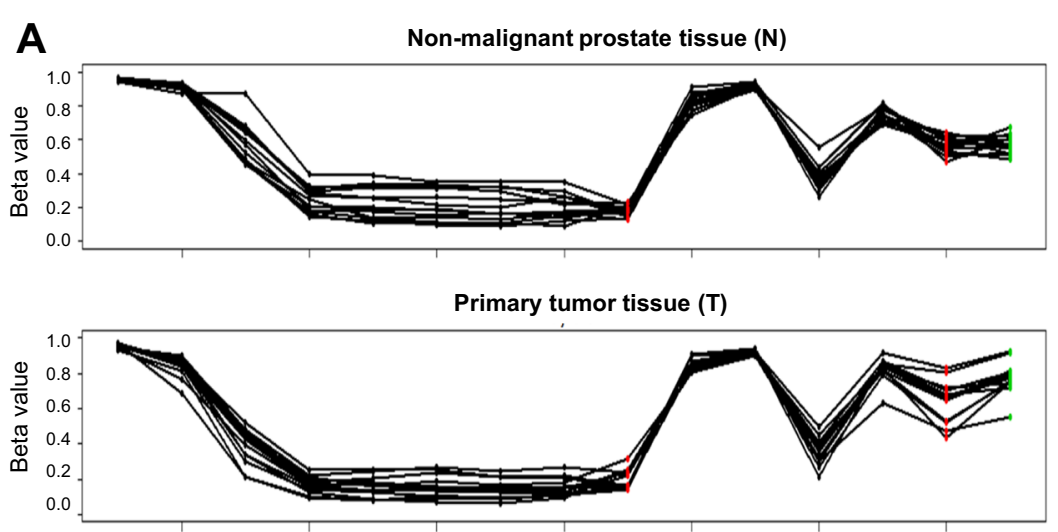

Metastasis (M), normal AR copy number (WT)

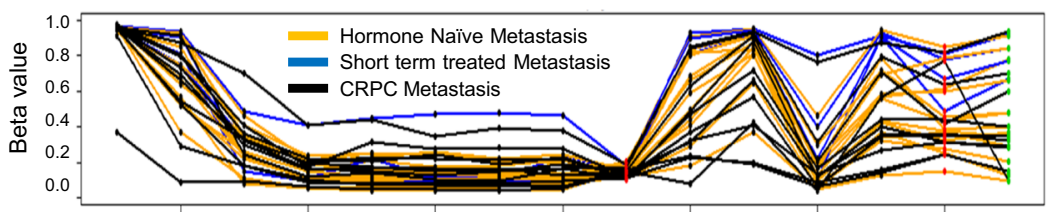

Metastasis (M), AR amplification

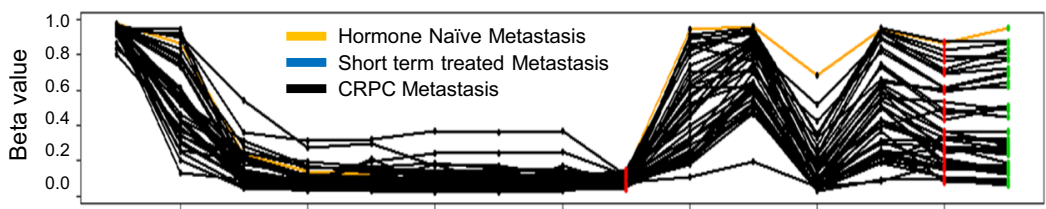

Mean N, T, M methylation

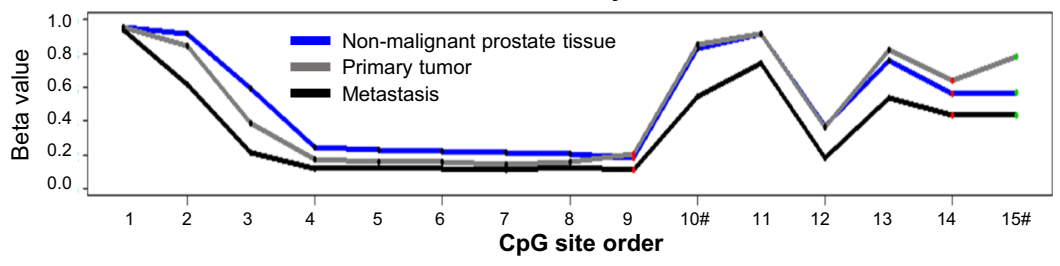

B

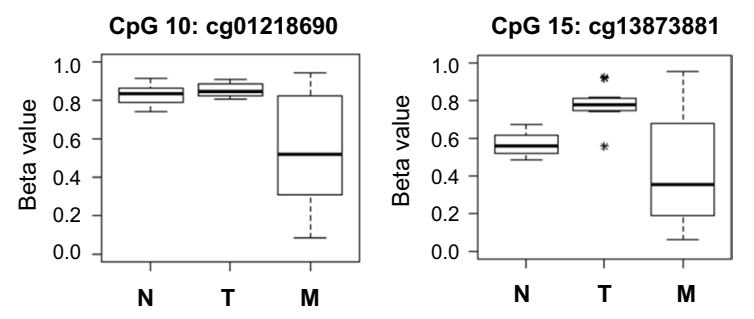

C
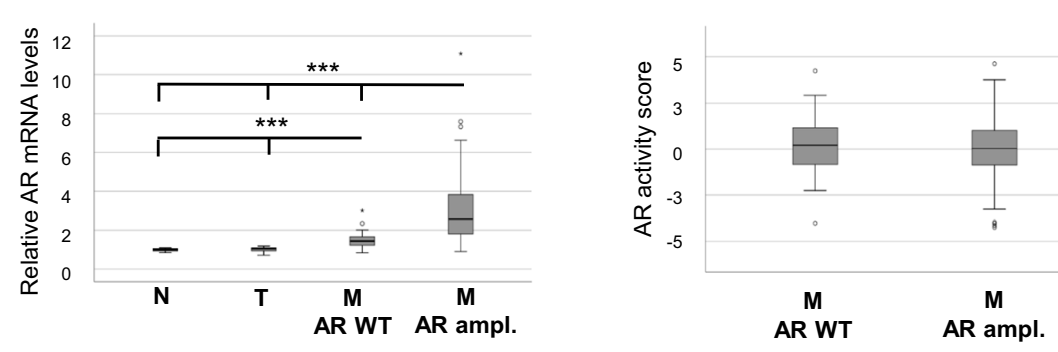

Fig. 3 (See legend on previous page.) 
methylation levels are related to the sample AR activity, defined from transcript levels of genes recently described to differentiate clinically relevant molecular subtypes of PC bone metastases based on AR activity $[9,10,12]$.

By pairwise comparisons of 12 tumor areas and adjacent non-malignant tissue isolated from radical prostatectomies, a pronounced hypermethylation was observed in primary PC. This is in line with a wealth of data, summarized in [15], suggesting not only that hypermethylation is an early event in PC tumorigenesis but also that it possibly could be used for diagnostic purpose. We observed hypermethylation of GSTP1, but also of other genes well known to be hypermethylated in PC, including AOX1, APC, BARHL2, CCDC8, CDKN2A, CYP27A1, EFS, GRASP, HOXA3, HOXC11, HOXD3, KIT, NXK2-1, NXK2-5, PHOX2A, POU3F3, PTGS2, RARB, RHCG, SIX6, TBX15, TMEM106A, WNT2, and ZNF154 [15] (Additional file 4: Table S1). Interestingly, methylation changes in APC, HOXD3, and PTGS2 have previously been suggested to provide prognostic information [2729]. The low number of primary tumor samples analyzed in the current study did, however, not allow evaluation of DM-CpGs in relation to prognosis. Instead, analysis was focused on identifying general effects of methylation on changes in gene expression during PC development. Functional pathway analysis using Gene Set Enrichment Analysis indicated hypermethylation and downregulation of genes associated with muscle contraction, such as ACTA, CNN, DES, TAGLN, and TPN, normally expressed in smooth muscle cells [30], but also of genes involved in diverse developmental (e.g. ANGPT1, STAT3, STAT5, TEK) and cytoskeletal (e.g. ANXA2, ANXA6, CD44, $S V I L)$ processes, as well as in regulation of proliferation (e.g. FGF2, FGFR1, PIK3R1, PRKCA) and cell adhesion (e.g. $C D H 5, C D H 23, P C D H 18, S T M N 2$ ). While reduced cell adhesion is an important hallmark for carcinomas, many of the other functional effects suggested from promoter hypermethylation in PC may be biased not only by the more abundant stroma in the $\mathrm{T}$ samples, but also by its different cellular content. While the stroma in the normal prostate is mainly composed of smooth muscle cells, prostate tumors show a progressive loss of smooth muscle cells in the favor of cancer-associated fibroblasts [31]. Hypomethylation in primary PC was uncommon and not associated with specific functional processes, at least based on the literature summarized by MetaCore ontology software.

Most of the identified DM-CpGs observed between primary $\mathrm{PC}$ and non-malignant prostate tissue were present also in the metastatic tissue. In addition, a unique set of DM-CpGs were observed when going from primary PC to metastases. The large majority of these were hypomethylated and included sites in the $A R$ promoter region. A detailed exploration showed a trend of demethylation at several CpGs in the $A R$ promoter, including two alternative transcription start sites that correlated well with induced levels of both $A R$ transcript 1 and 2 in the metastatic samples. High $A R$ mRNA levels in CRPC have been previously associated with $A R$ amplification [32-36], while methylation as a regulator of $A R$ expression is less well described and studies have also shown inconsistent results [16, 37-40]. Our results are in line with recent findings by Zaho and co-workers demonstrating $A R$ promoter hypomethylation in CRPC [16], but also add important knowledge by showing hypomethylation in previously un-treated metastasis samples suggesting that $A R$ demethylation may be an earlier cause to $A R$ induction in PC metastases than $A R$ amplification, as $A R$ amplification is generally not observed prior to ADT. Importantly, neither the large variance in $A R$ promoter methylation levels observed among the metastatic samples nor their $A R$ amplification status showed any clear relationship to the tumor sample AR activity.

In an attempt to study universal promoter methylation in relation to AR activity within individual metastasis samples, each sample was given an AR activity score related to its expression levels of genes involved in or regulated by canonical AR signaling, and $\mathrm{mCpGs}$ positively or negatively correlated to this score were taken further for functional exploration. A Methylation Classifier for Androgen receptor activity (MCA) signature was identified and used to classify the bone metastasis samples. Taken together, our results suggested that low promoter methylation levels of certain AR-regulated genes (i.e. SLC45A3, STEAP2, ELK4 and TMPRSS2) may contribute to the AR-driven tumor phenotype seen in the majority of CRPC patients with metastatic disease [2-12], while high promoter methylation of the same genes may contribute

\footnotetext{
(See figure on next page.)

Fig. 4 The promoter Methylation Classifier for Androgen receptor activity (MCA) signature in relation to tumor and patient characteristics. A Heat map showing the 4580 CpG sites in the MCA signature which correlated to AR activity score (corr $>0.4$ or $<-0.4$, StDev $>0.15)$ in 66 bone metastases from PC. Primary tumors $(n=12)$ and non-malignant prostate tissue $(n=12)$ are shown as reference. MCA negative and MCA positive metastasis are marked in the figure. Colored bars show sample characteristics with respect to patient treatment status, tissue composition, MetA-C class, and AR copy number. Integrated expression analysis and enriched pathways for genes in cluster A-E are shown in Table 2. B AR activity scores of MCA positive and MCA negative bone metastasis. $\mathbf{C}$ Proliferation scores of the MCA positive and MCA negative bone metastasis samples. D Serum PSA levels of MCA positive and MCA negative patients with metastases. E Kaplan Meier survival analysis of MCA positive and MCA negative patients with metastases. The AR and proliferation scores are defined and calculated as described in the "Material and methods" section
} 


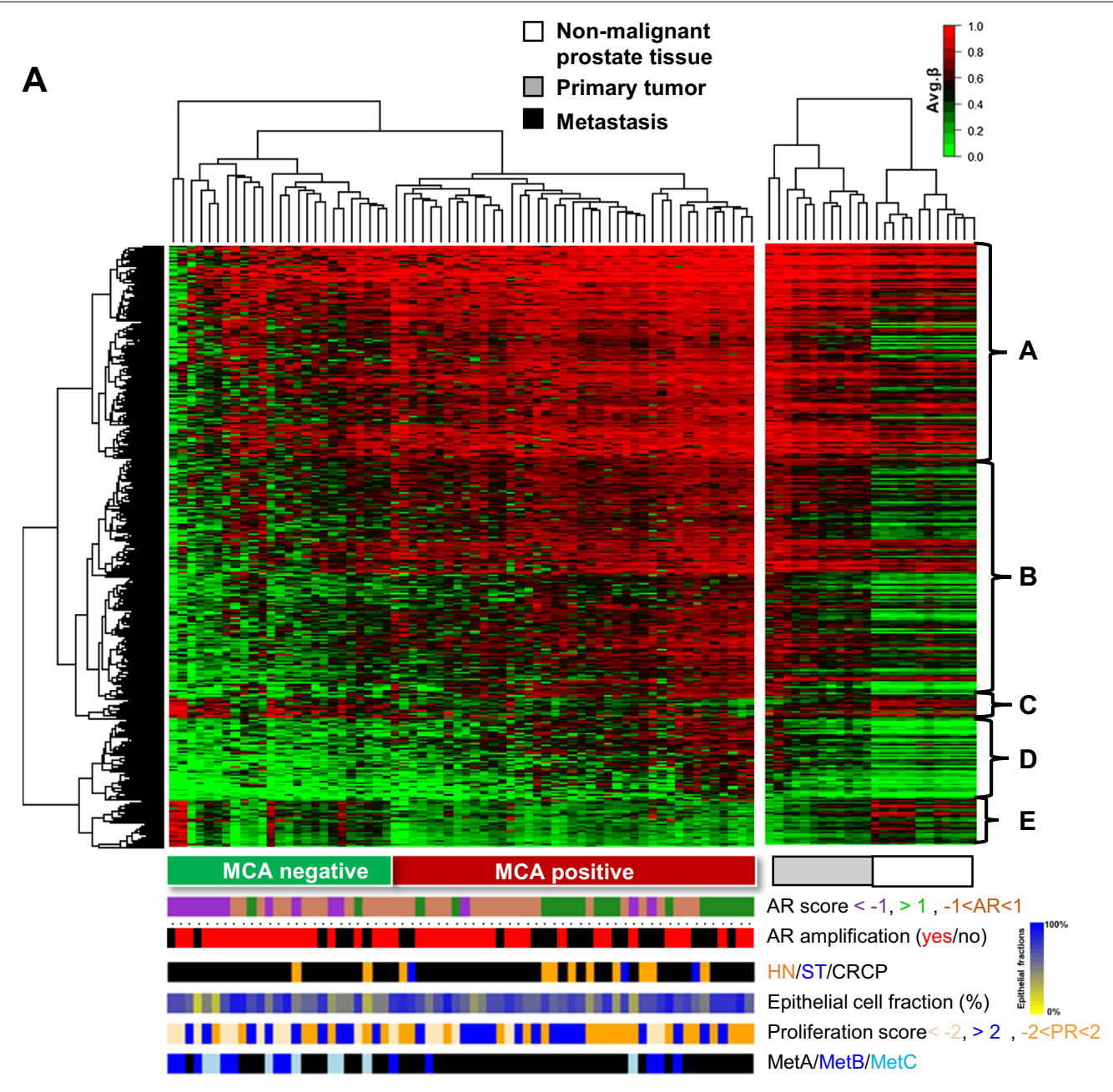

B

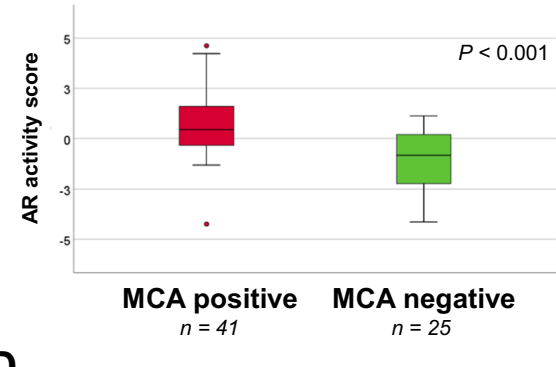

D

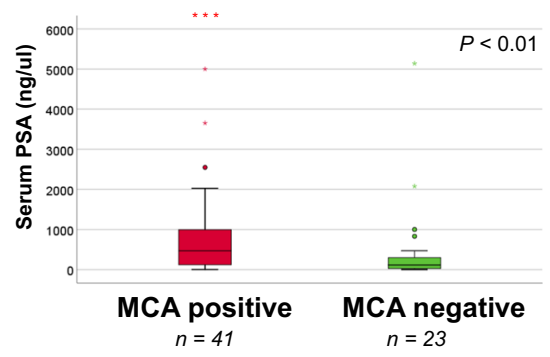

C

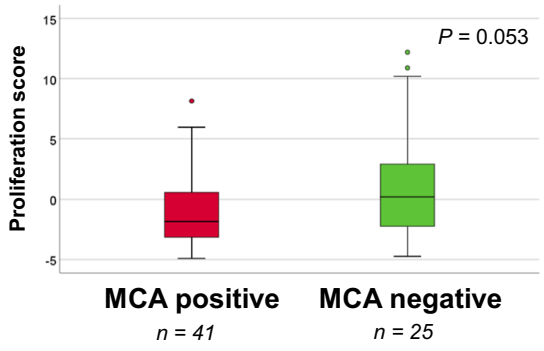

E

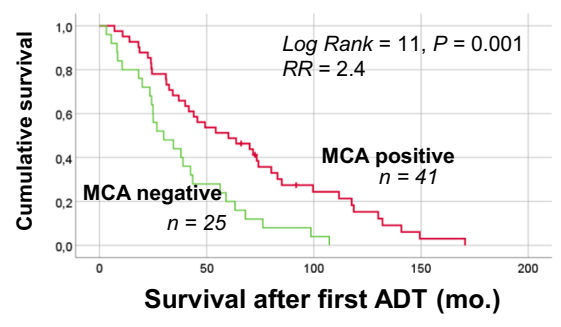

Fig. 4 (See legend on previous page.) 
Table 2 Genes corresponding to cluster A-E in Fig. 4A showing hyper- (A, B, D) or hypomethylation (C, E) in relation to AR activity in bone metastases, and functionally enriched pathways according to MetaCore analysis of genes with inverse correlation between CpG methylation and gene expression

\begin{tabular}{|c|c|c|}
\hline Cluster & Genes & Enriched pathways \\
\hline A & $\begin{array}{l}\text { ABCB9, ACOT11, ADAM28, ATHL1, AVIL, B3GNT5, BAALC, BATF, } \\
\text { C14ORF173, C20ORF118, CCDC109B, CD300LF, CD38, CD5, CDC25B, } \\
\text { CNPY4, CTLA4, E2F2, ENTPD3, FAM110B, GPR68, HCST, HEY1, HOXB4, } \\
\text { HOXB5, HOXB6, INF2, KCNJ5, KIAA1274, KLHL2, KLK12, LIMS1, } \\
\text { MAP1LC3A, MARCH3, MCOLN3, MDC1, MST1R, MXI1, NHS, NME2, } \\
\text { NNT, NPL, OAS2, PARVB, PARVG, PAX6, PHF11, PIK3CD, PKM2, PLEKHO1, } \\
\text { PPAP2C, PTGER1, RALA, RBM38, RBP1, ROD1, RPH3AL, RUNX2, SCNN1A, } \\
\text { SCRN1, SDPR, SH2B3, SKA2, SLC43A3, SPC25, SPOCK2, SYK, TCF4, TCP11, } \\
\text { TMEM105, TMEM176A, TNS3, TOX3, TRIM9, TYMS, UCHL1, WASPIP, VDR, } \\
\text { VIM, YWHAH, ZBP1 }\end{array}$ & \multirow[t]{3}{*}{$\begin{array}{l}\text { Development: TGF-beta-dependent induction of EMT via RhoA, PI3K } \\
\text { and ILK } \\
\text { Cytoskeleton remodeling: Reverse signaling by Ephrin-B, Integrin } \\
\text { outside-in signaling } \\
\text { Immune response: IL-15 signaling via JAK-STAT cascade, KLRK1 } \\
\text { (NKG2D) signaling pathway, IL-2 signaling via JAK STAT, Role of } \\
\text { DAP12 receptors in NK cells } \\
\text { CHDI_Correlations from discovery data causal network } \\
\text { SHH signaling in colorectal cancer }\end{array}$} \\
\hline B & $\begin{array}{l}\text { ABP1, ACOT11, ACTB, ARHGEF2, BATF, C6ORF115, CCDC28B, CCR1, } \\
\text { CD300LF, CD34, COL4A6, CX3CL1, ELF4, EMP1, EPHA2, FBXL10, FGR, } \\
\text { FOXM1, GADD45A, GALE, GIMAP8, GMFG, HES6, HMGB3, HOXB3, } \\
\text { HOXB4, HOXB6, IFI27, IL23A, IL2RB, IVNS1ABP, KLF13, KLHL6, LBX2, } \\
\text { LRRN2, MAL, NCKAP1L, NDUFA4L2, NPAS2, NRM, NT5DC2, PAM, PARVG, } \\
\text { PCK2, PIGR, PITX1, PPT2, RFXDC2, RNF34, SARDH, SDC1, SEL1L3, SER- } \\
\text { PINB1, SHANK3, SLC15A3, SLC35D3, SOX2, SPOCK2, STAT5A, SULT1A1, } \\
\text { TAGLN2, TCF4, TIMP1, TMEM105, TMEM173, TOX3, TYROBP }\end{array}$ & \\
\hline $\mathrm{D}$ & $\begin{array}{l}\text { AGPS, BTG1, CD93, CENPV, GNAI2, LRFN4, NCAPG, NCF4, PRR7, PTK6, } \\
\text { SC65, SDC1, SGOL1, TAGLN2, TGIF2, TMEM37, TNFAIP8L1, TSPAN4, } \\
\text { UCP2 }\end{array}$ & \\
\hline $\bar{C}$ & $\begin{array}{l}\text { ACSL3, BBS4, C10ORF81, CANT1, CREB3L4, CTDSP1, EHF, GREB1, MLPH, } \\
\text { NDRG3, NEDD4L, NPAL3, NUP93, RPL37, SGMS1, STEAP2, SVIL }\end{array}$ & \multirow{2}{*}{$\begin{array}{l}\text { Recurrent gene fusions in prostate cancer } \\
\text { Transcription targets of androgen receptor involved in prostate cancer } \\
\text { Androgen receptor activation and downstream signaling in prostate } \\
\text { cancer } \\
\text { TMPRSS2-ERG fusion in prostate cancer } \\
\text { G-protein signaling_G-protein alpha-i signaling cascades } \\
\text { Signal transduction_mTORC1 downstream signaling }\end{array}$} \\
\hline E & $\begin{array}{l}\text { ATP6A, C19ORF48, CANT1, CLIP1, EHF, ELK4, GNL3, KIAA1244, NEDD4L, } \\
\text { PEX11A, RAP1GAP, RGS10, SLC45A3, SPDF, STX19, SVIL, TMPRSS2, } \\
\text { TRIM36, XPO6, ZNF280D }\end{array}$ & \\
\hline
\end{tabular}

to the development of less AR-dependent CRPC, commonly referred to as AR-indifferent, small-cell or neuroendocrine-like CRPC [41]. It is of importance to note, though, that histological evaluation did not confirm any enrichment of a neuroendocrine-like phenotype in samples of the MCA negative compared to the positive cluster. This was concluded from reviewing the results from immunohistochemical analysis of the neuroendocrine marker chromogranin A, previously assessed in 60 of the 66 MCA-classified samples [12] (data not shown). Neither could the MCA class be predicted from immunohistochemical analysis of the AR protein level [12] $(n=63$, data not shown).

Samples of the metastasis subtype MetA previously described by us as AR-driven [12] primarily showed hypomethylation and high expression of canonically AR-regulated genes, while samples of the less AR-driven subtypes MetB and MetC were enriched among samples with an MCA negative signature (showing hypermethylation and low expression of many AR targeting genes). Indirectly, our result thus implies promoter methylation as a possible mechanism behind the low AR activity defining metastasis subtypes MetB and MetC. Accordingly, patients with MCA positive signature had higher serum PSA levels and a better prognosis than the smaller group of MCA negative patients, probably due to more differentiated, less proliferative tumor cells being more AR dependent and thereby showing a better response to AR targeting therapies, similar to what previously have been described for MetA in comparison to MetB-C [12]. Notably, the MCA signature provided strong information related to patient prognosis after ADT, with MCA positive patients showing 2-times longer median survival than MCA negative patients, resulting in a 5-year cancerspecific survival of 50 and $20 \%$ respectively. Despite the obvious clinical relevance of separating metastasis samples into MCA positive or negative cases, the clusters showed variable consistencies according to the silhouette analysis. This might originate from the procedure of selecting all $\mathrm{mCpG}$ sites for analysis that correlated to the tumor AR activity on a continuous scale, i.e. the classifier was not originally built to dichotomize samples into two groups.

As MCA negative metastases with low AR activity scores were specified by hypermethylation of AR-regulated genes, it is tempting to speculate that they could be sensitized for AR-directed therapies by treatment with demethylating agents, such as have been shown possible 
Table 3 Univariate and multivariate Cox regression survival analysis of MCA cluster and other characteristics of interest, in relation to cancer-specific survival after androgen-deprivation therapy

\begin{tabular}{lllr}
\hline & $P$ & $R \boldsymbol{R}$ & $\mathbf{9 5 \%} \mathbf{C l}$ \\
\hline Univariate & & & \\
MCA cluster (negative vs. positive, $n=25$ & $\mathbf{0 . 0 0 1 6}$ & 2.4 & $1.4-4.1$ \\
$\quad$ and 41) & & & \\
AR score & 0.35 & 0.9 & $0.8-1.1$ \\
Prol. score & $\mathbf{0 . 0 3 9}$ & 1.1 & $1.0-1.1$ \\
AR (ampl. vs. WT, $n=24$ and 42) & 0.12 & 1.5 & $0.9-2.6$ \\
Age (at metastsis surgery) & 0.42 & 1.0 & $1.0-1.0$ \\
Metastasis subtype & & & \\
$\quad$ MetA ( $n=46)$ & & 1.0 & \\
$\quad$ MetB ( $n=12)$ & $\mathbf{0 . 0 3 4}$ & 2.0 & $1.1-3.9$ \\
$\quad$ MetC ( $n=8)$ & 0.95 & 0.98 & $0.45-2.1$ \\
Serum PSA (at metastasis surgery) & 0.07 & 1.0 & $1.0-1.0$ \\
Multivariate & & & \\
MCA cluster (negative vs. positive, $n=25$ & $\mathbf{0 . 0 0 1 7}$ & 2.7 & $1.5-5.1$ \\
$\quad$ and 41) & & & \\
Prol. score & 0.89 & 0.99 & $0.90-1.1$ \\
Metastasis subtype & & 1.0 & \\
$\quad$ MetA ( $n=46)$ & & 1.6 & $0.52-5.2$ \\
MetB $(n=12)$ & 0.40 & 0.58 & $0.25-1.3$ \\
MetC ( $n=8$ ) & 0.20 & & \\
\hline
\end{tabular}

Variables with a P-value below 0.05 (shown in bold) in univariate survival analysis were taken further into multivariate analysis

" Metastasis subtypes according to [12]

by inhibitors to DNA methyl transferase 1 in different experimental systems for CRPC [42, 43]. In a recent paper, Xiao and co-workers furthermore identified the histone lysine- $N$-methyltransferase EZH2 as a possible epigenetic regulator of tumor response to AR-targeting therapy and demonstrated possibilities with its inhibition in the treatment of CRPC [44].

While the methylation levels of a relatively small set of genes were directly associated with the AR activity observed in PC bone metastases, the methylation level of a much larger gene set seemed involved in regulating diverse processes such as EMT, cytoskeletal remodeling, and immune responses, all standing in an inverse correlation to tumor AR activity.

In conclusion, this study describes general patterns of gene promoter methylation during PC disease progression, and specifically identifies a novel promoter MCA signature that is associated with canonical AR activity in PC bone metastases and to patient prognosis after ADT. Based on this methylation signature, patients with PC bone metastases could be stratified into one of two patient groups; MCA positive patients being likely to respond to ADT and possibly also other AR targeting therapies or MCA negative patients being less responsive to AR targeting therapies, but possibly suitable for treatment with AR inhibitors in combination with epigenetic modulators (currently in clinical trials, https:// clinicaltrials.gov). The next step will be to verify the suggested therapy-predictive value of the MCA signature. This could be retrospectively done by analyzing methylation signatures in circulating tumor DNA collected from patients prior to treatment for metastatic PC, similarly to what is described in a recent paper by $\mathrm{Wu}$ et al. [45]. For clinical implementation, however, a stable non-cluster-based classifier need to be developed. The overall cause to the two methylation types observed among PC bone metastases also remains to be identified, as it could give important implications for future developments of novel treatment strategies.

\section{Abbreviations}

AR: Androgen receptor; ADT: Androgen-deprivation therapy; $\beta$-value: Methylation level; CNV: Copy number variation; CRPC: Castration-resistant prostate cancer; DM: Differentially methylated; EMT: Epithelial to mesenchymal transition; HN: Hormone-naïve; M: Metastasis tissue; MCA: Methylation classifier for androgen receptor activity; $\mathrm{mCpGs}$ : Methylation levels ( $\beta$ values) of $\mathrm{CpG}$ sites; MetA-C: Metastasis subtype A-C; meQTL: Methylation quantitative trait loci; $\mathrm{N}$ : Non-malignant prostate tumor tissue; PC: Prostate cancer; PCA: Principal component analysis; PSA: Prostate specific antigen; SNP: Single nucleotide polymorphism; ST: Short-term castrated; T: Malignant prostate tumor tissue; TSS: Transcription start site.

\section{Supplementary Information}

The online version contains supplementary material available at https://doi. org/10.1186/s13148-021-01119-0.

Additional file 1: Fig. S1. Analysis of 59 built-in SNP on the HumanMethylation EPIC array to confirm identity of multiple samples taken from the same individual ( $\mathrm{N}$ and $\mathrm{T}$ tissue).

Additional file 2: Fig. S2. Schematic flowchart of the pre-processing steps of the HumanMethylation EPIC arrays.

Additional file 3: Fig. S3. Silhouette analysis showing the cluster consistencies of the MCA positive and negative clusters in Figure 4A.

Additional file 4: Table S1. Differetially methylated CpGs in promoter regions of genes in primary prostate tumor tissue $(T)$ compared to adjacent non-malignant prostate tissue $(\mathrm{N})$, their correlations to corresponding gene expression levels, and the fold change in gene expression levels between sample groups.

Additional file 5: Table S2. Top 10 enriched process networks in localized prostate tumor tissue ( $\mathrm{T}$ ) compared to adjacent non-malignant prostate tissue $(\mathrm{N})$ or in prostate cancer bone metastases $(\mathrm{M})$ compared to localized tumors.

Additional file 6: Table S3. Differetially methylated CpGs in promoter regions of genes in bone metastases $(M)$ compared to localized prostate tumors $(T)$, their correlations to corresponding gene expression levels, and the fold change in gene expression levels between sample groups.

Additional file 7: Table S4. CpG sites of the AR gene shown in Figure 3A. 


\section{Acknowledgements}

Pernilla Andersson and Susanne Gidlund are acknowledged for superior technical assistance.

\section{Authors' contributions}

PW and SD conceived and designed the study. EBY, EJ and MB performed experiments. ET, ML, and PW analyzed the data. EBY, SD, PW wrote the first draft of the manuscript. SC and AW collected biological samples and clinical data. AB performed histological analysis. MH supervised the study. All authors read and approved the final manuscript.

\section{Funding}

Open access funding provided by Umea University. The study was supported by grants from the Cancerfonden, Kempestiftelserna, Swedish Research Council, the Swedish Cancer Society, Swedish Foundation for Strategic Research, Cancer Research Foundation in Northern Sweden, the Kempe research foundation, The Swedish Prostate Cancer Federation, the Medical Faculty of Umeå University, and Uppsala-Umeå Comprehensive Cancer Consortium. Financial support was also provided through a regional agreement between Umeå University and Västerbotten County Council on cooperation in the field of Medicine, Odontology and Health.

\section{Availability of data and materials}

The dataset generated (GSE174613) and analyzed (GSE29650 and GSE101607) during the current study are available at https://www.ncbi.nlm.nih.gov/geo/.

\section{Declarations}

\section{Ethics approval and consent to participate}

Patients gave their informed consent and the study was conducted in accordance with the Declaration of Helsinki. The study was approved by the local ethic review board of Umeå University (Dnr 03-158, Dnr 04-26M, Dnr 03-482, Dnr 2013-57-31M, Dnr 2017-197-32M).

\section{Consent for publication}

$$
\text { Not applicable. }
$$

\section{Competing interests}

The authors declare that they have no competing interests.

\section{Author details}

'Department of Medical Biosciences, Pathology, Umeå University, Umeå, Sweden. ${ }^{2}$ Department of Surgical and Perioperative Sciences, Orthopedics, Umeå University, Umeå, Sweden. ${ }^{3}$ Department of Radiation Sciences, Oncology, Umeå University, Umeå, Sweden. ${ }^{4}$ Department of Clinical Microbiology, Umeå University, Umeå, Sweden.

Received: 8 February 2021 Accepted: 20 June 2021

Published online: 30 June 2021

\section{References}

1. Cornford P, van den Bergh RCN, Briers E, Van den Broeck T, Cumberbatch MG, De Santis M, et al. EAU-EANM-ESTRO-ESUR-SIOG guidelines on prostate cancer. Part II-2020 update: treatment of relapsing and metastatic prostate cancer. Eur Urol. 2020;20:30773-9.

2. Robinson D, Van Allen EM, Wu YM, Schultz N, Lonigro RJ, Mosquera JM, et al. Integrative clinical genomics of advanced prostate cancer. Cell. 2015;161:1215-28.

3. Quigley DA, Dang HX, Zhao SG, Lloyd P, Aggarwal R, Alumkal JJ, et al. Genomic hallmarks and structural variation in metastatic prostate cancer. Cell. 2018;175:889.

4. De Laere B, Oeyen S, Mayrhofer M, Whitington T, van Dam PJ, Van Oyen P, et al. TP53 outperforms other androgen receptor biomarkers to predict abiraterone or enzalutamide outcome in metastatic castration-resistant prostate cancer. Clin Cancer Res. 2019;25:1766-73.

5. Abida W, Cyrta J, Heller G, Prandi D, Armenia J, Coleman I, et al. Genomic correlates of clinical outcome in advanced prostate cancer. Proc Natl Acad Sci U S A. 2019;116:11428.
6. De Laere B, van Dam PJ, Whitington T, Mayrhofer M, Diaz EH, Van den Eynden $\mathrm{G}$, et al. Comprehensive profiling of the androgen receptor in liquid biopsies from castration-resistant prostate cancer reveals novel intra-AR structural variation and splice variant expression patterns. Eur Urol. 2017;72:192-200.

7. Hörnberg E, Ylitalo EB, Crnalic S, Antti H, Stattin P, Widmark A, Bergh A, Wikström $P$. Expression of androgen receptor splice variants in prostate cancer bone metastases is associated with castration-resistance and short survival. PLoS ONE. 2011;6:e19059.

8. Jernberg E, Thysell E, Bovinder Ylitalo E, Rudolfsson S, Crnalic S, Widmark A, Bergh A, Wikström P. Characterization of prostate cancer bone metastases according to expression levels of steroidogenic enzymes and androgen receptor splice variants. PLoS ONE. 2013;8:e77407.

9. Ylitalo EB, Thysell E, Jernberg E, Lundholm M, Crnalic S, Egevad L, et al. Subgroups of castration-resistant prostate cancer bone metastases defined through an inverse relationship between androgen receptor activity and immune response. Eur Urol. 2017;71:776-87.

10. Nordstrand A, Bovinder Ylitalo E, Thysell E, Jernberg E, Crnalic S, Widmark $A$, et al. Bone cell activity in clinical prostate cancer bone metastasis and its inverse relation to tumor cell androgen receptor activity. Int J Mol Sci. 2018;19:1223.

11. Iglesias-Gato D, Thysell E, Tyanova S, Crnalic S, Santos A, Lima TS, et al. The proteome of prostate cancer bone metastasis reveals heterogeneity with prognostic implications. Clin Cancer Res. 2018;24:5433-44.

12. Thysell E, Vidman L, Ylitalo EB, Jernberg E, Crnalic S, Iglesias-Gato D, et al. Gene expression profiles define molecular subtypes of prostate cancer bone metastases with different outcomes and morphology traceable back to the primary tumor. Mol Oncol. 2019;13:1763.

13. You S, Knudsen BS, Erho N, Alshalalfa M, Takhar M, Al-Deen Ashab H, et al. Integrated classification of prostate cancer reveals a novel luminal subtype with poor outcome. Cancer Res. 2016;76:4948.

14. Zhao SG, Chang SL, Erho N, Yu M, Lehrer J, Alshalalfa M, et al. Associations of luminal and basal subtyping of prostate cancer with prognosis and response to androgen deprivation therapy. JAMA Oncol. 2017;3:1663.

15. Massie CE, Mills IG, Lynch AG. The importance of DNA methylation in prostate cancer development. J Steroid Biochem Mol Biol. 2017;166:1-15.

16. Zhao SG, Chen WS, Li H, Foye A, Zhang M, Sjöström M, et al. The DNA methylation landscape of advanced prostate cancer. Nat Genet. 2020;52:778-89.

17. Pomerantz MM, Qiu X, Zhu Y, Takeda DY, Pan W, Baca SC, et al. Prostate cancer reactivates developmental epigenomic programs during metastatic progression. Nat Genet. 2020;52:790-9.

18. Borssén M, Haider Z, Landfors M, Norén-Nyström U, Schmiegelow K, Åsberg AE, et al. DNA methylation adds prognostic value to minimal residual disease status in pediatric T-cell acute lymphoblastic leukemia. Pediatr Blood Cancer. 2016;63:1185-92.

19. Teschendorff AE, Marabita F, Lechner M, Bartlett T, Tegner J, GomezCabrero D, Beck S. A beta-mixture quantile normalization method for correcting probe design bias in Illumina Infinium 450 k DNA methylation data. Bioinformatics. 2013;29:189-96.

20. Gaunt TR, Shihab HA, Hemani G, Min JL, Woodward G, Lyttleton O, et al. Systematic identification of genetic influences on methylation across the human life course. Genome Biol. 2016;17:61.

21. McClay JL, Shabalin AA, Dozmorov MG, Adkins DE, Kumar G, Nerella S, Clark SL, Bergen SE, Swedish Schizophrenia Consortium, Hultman CM, Magnusson PK, Sullivan PF, Aberg KA, van den Oord EJ. High density methylation QTL analysis in human blood via next-generation sequencing of the methylated genomic DNA fraction. Genome Biol. 2015;16:291.

22. Zhou W, Laird PW, Shen H. Comprehensive characterization, annotation and innovative use of Infinium DNA methylation BeadChip probes. Nucleic Acids Res. 2017;45(4):22.

23. Aryee MJ, Jaffe AE, Corrada-Bravo H, Ladd-Acosta C, Feinberg AP, Hansen KD, et al. Minfi: a flexible and comprehensive Bioconductor package for the analysis of Infinium DNA methylation microarrays. Bioinformatics. 2014;30:1363-9.

24. Hovestadt V, Zapatka M. conumee: enhanced copy-number variation analysis using Illumina DNA methylation arrays. R package version 1.9.0, http://bioconductor.org/packages/conumee/.

25. Cuzick J, Swanson GP, Fisher G, Brothman AR, Berney DM, Reid JE, et al. Prognostic value of an RNA expression signature derived from cell cycle 
proliferation genes in patients with prostate cancer: a retrospective study. Lancet Oncol. 2011;12:245.

26. Maechler M, Rousseeuw P, Struyf A, Hubert M, Hornik K. cluster: cluster analysis basics and extensions. R package version 2.1.0. 2019 (2021).

27. Yegnasubramanian S, Kowalski J, Gonzalgo ML, Zahurak M, Piantadosi S, Walsh PC, et al. Hypermethylation of CpG islands in primary and metastatic human prostate cancer. Cancer Res. 2004;64:1975-86.

28. Henrique R, Ribeiro FR, Fonseca D, Hoque MO, Carvalho AL, Costa VL, et al. High promoter methylation levels of APC predict poor prognosis in sextant biopsies from prostate cancer patients. Clin Cancer Res. 2007;13:6122-9.

29. Stott-Miller M, Zhao S, Wright JL, Kolb S, Bibikova M, Klotzle B, et al. Validation study of genes with hypermethylated promoter regions associated with prostate cancer recurrence. Cancer Epidemiol Biomark Prev. 2014;23:1331-9.

30. Lee MY, Park C, Berent RM, Park PJ, Fuchs R, Syn H, et al. Smooth muscle cell genome browser: enabling the identification of novel serum response factor target genes. PLoS ONE. 2015;10(8):e0133751.

31. Cunha GR, Hayward SW, Wang YZ, Ricke WA. Role of the stromal microenvironment in carcinogenesis of the prostate. Int J Cancer. 2003:107:1-10.

32. Visakorpi T, Hyytinen E, Koivisto P, Tanner M, Keinanen R, Palmberg C, et al. In vivo amplification of the androgen receptor gene and progression of human prostate cancer. Nat Genet. 1995;9:401-6.

33. Palmberg C, Koivisto P, Hyytinen E, Isola J, Visakorpi T, Kallioniemi OP, Tammela T. Androgen receptor gene amplification in a recurrent prostate cancer after monotherapy with the nonsteroidal potent antiandrogen Casodex (bicalutamide) with a subsequent favorable response to maximal androgen blockade. Eur Urol. 1997;31:216-9.

34. Bubendorf L, Kononen J, Koivisto P, Schraml P, Moch H, Gasser TC, et al. Survey of gene amplifications during prostate cancer progression by high-throughout fluorescence in situ hybridization on tissue microarrays. Cancer Res. 1999;59:803-6.

35. Kaltz-Wittmer C, Klenk U, Glaessgen A, Aust DE, Diebold J, Lohrs U, Baretton GB. FISH analysis of gene aberrations (MYC, CCND1, ERBB2, RB, and $A R$ ) in advanced prostatic carcinomas before and after androgen deprivation therapy. Lab Investig J Tech Methods Pathol. 2000;80:1455-64.

36. Djusberg E, Jernberg E, Thysell E, Golovleva I, Lundberg P, Crnalic S, et al. High levels of the AR-V7 splice variant and co-amplification of the golgi protein coding YIPF6 in AR amplified prostate cancer bone metastases. Prostate. 2017;77:625-38.

37. Kinoshita H, Shi Y, Sandefur C, Meisner LF, Chang C, Choon A, et al. Methylation of the androgen receptor minimal promoter silences transcription in human prostate cancer. Cancer Res. 2000;60:3623-30.

38. Nakayama T, Watanabe M, Suzuki H, Toyota M, Sekita N, Hirokawa Y, et al. Epigenetic regulation of androgen receptor gene expression in human prostate cancers. Lab Investig. 2000;80:1789-96.

39. Friedlander TW, Roy R, Tomlins SA, Ngo VT, Kobayashi Y, Azameera A, et al. Common structural and epigenetic changes in the genome of castrationresistant prostate cancer. Cancer Res. 2012;72:616-25.

40. Jarrard DF, Kinoshita H, Shi Y, Sandefur C, Hoff D, Meisner LF, et al. Methylation of the androgen receptor promoter $\mathrm{CpG}$ island is associated with loss of androgen receptor expression in prostate cancer cells. Cancer Res. 1998:58:5310-4.

41. Beltran H, Prandi D, Mosquera JM, Benelli M, Puca L, Cyrta J, et al. Divergent clonal evolution of castration-resistant neuroendocrine prostate cancer. Nat Med. 2016;22:298-305.

42. Gravina GL, Festuccia C, Millimaggi D, Dolo V, Tombolini V, de Vito M, Vicentini C, Bologna M. Chronic azacitidine treatment results in differentiating effects, sensitizes against bicalutamide in androgen-independent prostate cancer cells. Prostate. 2008;68:793-801.

43. Gravina GL, Marampon F, Di Staso M, Bonfili P, Vitturini A, Jannini EA, et al. 5-Azacitidine restores and amplifies the bicalutamide response on preclinical models of androgen receptor expressing or deficient prostate tumors. Prostate. 2010;70:1166-78.

44. Xiao L, Tien JC, Vo J, Tan M, Parolia A, Zhang Y, et al. Epigenetic reprogramming with antisense oligonucleotides enhances the effectiveness of androgen receptor inhibition in castration-resistant prostate cancer. Cancer Res. 2018;78:5731-40.

45. Wu A, Cremaschi P, Wetterskog D, Conteduca V, Franceschini GM, Kleftogiannis D, et al. Genome-wide plasma DNA methylation features of metastatic prostate cancer. J Clin Investig. 2020;130:1991-2000.

\section{Publisher's Note}

Springer Nature remains neutral with regard to jurisdictional claims in published maps and institutional affiliations.
Ready to submit your research? Choose BMC and benefit from:

- fast, convenient online submission

- thorough peer review by experienced researchers in your field

- rapid publication on acceptance

- support for research data, including large and complex data types

- gold Open Access which fosters wider collaboration and increased citations

- maximum visibility for your research: over $100 \mathrm{M}$ website views per year

At BMC, research is always in progress.

Learn more biomedcentral.com/submissions 